\title{
LINE SEGMENTS IN THE ISOTROPIC PLANAR STIT TESSELLATION
}

\author{
RICHARD COWAN, ${ }^{*}$ University of Sydney
}

\begin{abstract}
This paper presents a powerful characterisation for the structure of internal vertices of the STIT's $I$-segments. The characterisation allows certain mathematical analyses to be performed easily. We demonstrate this by deriving new results for various topological properties of the tessellation: for example, the numbers of various types of edge and cell side within the typical $I$-segment. The characterisation also provides a tool for the calculations of metric properties of the tessellation; many new length distributions and frame-coverage results are given.
\end{abstract}

Keywords: Random tessellation; STIT tessellation; stochastic geometry

2010 Mathematics Subject Classification: Primary 60D05

Secondary 60G55

\section{Introduction}

The planar STIT tessellation, first introduced by Nagel and Weiss [8], [9] and studied by them and others (see [5], [6], [7], [12], and [14]), has in recent years become one of the important stationary tessellation models. In this paper we present a characterisation for the structure of internal vertices of the STIT's so-called I-segments.

Figure 1 shows a realisation of an isotropic STIT tessellation. It contains some line segments that, taken together with small neighbourhoods around each end, look like an uppercase $I$. These are the $I$-segments. Formally, an $I$-segment in a STIT tessellation is a convex union of tessellation edges that is not contained in a longer convex union of edges. A convex union is, of course, a union of collinear edges (which join together to make the line segment). (Terminology: A stationary tessellation is a partition of the plane into convex polygonal cells. Each cell has sides and corners, these being respectively the 1 -faces and 0 -faces of the polygon. The union (taken over all cells) of the cell's corners is a collection of points in the plane called the vertices of the tessellation. Those line segments which have a vertex at each end and no vertices in their interior are called edges of the tessellation.)

The new characterisation presented below in Theorem 1 enables computation of the probability distributions for the numbers of various types of edge and cell side within the typical $I$-segment and the number of vertices internal to a typical cell side. It also allows us to address metric issues concerning the tessellation's frame and its $I$-segments, cell sides, and edges; for example, we find the proportion of the frame which is covered by certain types of edge and the length distribution of an edge given that it is of a particular type. Furthermore, Theorem 1 allows us to find some adjacency results (for example, the mean number of cell sides adjacent to a typical cell side) that have, to date, proved elusive. (Terminology: Two geometric elements $x$ and $y$ are said to be adjacent if either $x \subseteq y$ or $y \subseteq x$. The frame of

Received 20 October 2011; revision received 2 July 2012.

* Postal address: School of Mathematics and Statistics, University of Sydney, NSW, 2006, Australia.

Email address: rcowan@usyd.edu.au 


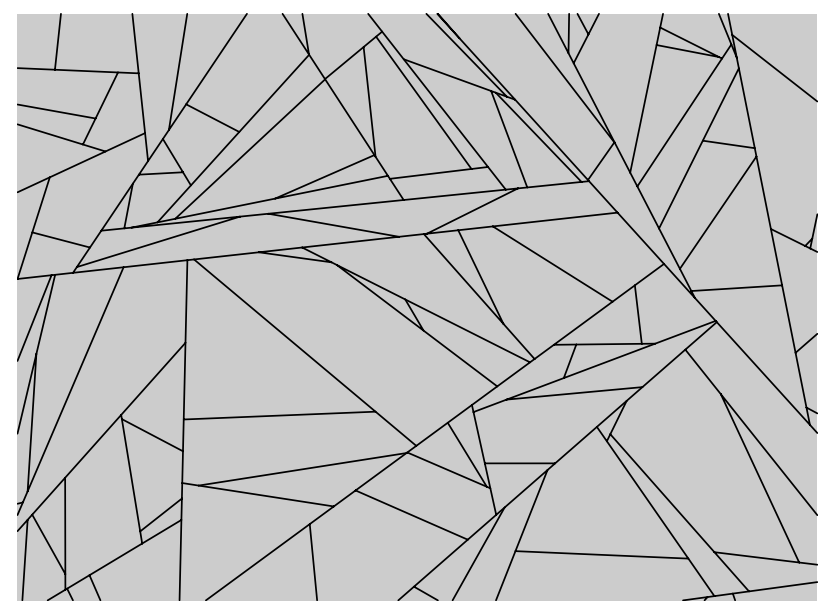

FIGURE 1: A realisation of the STIT model within a rectangular viewing window.

a tessellation is the union of all its edges. The frame of a STIT tessellation which has frame intensity $t$ is called $Y(t)$. As with all stationary random structures, a 'typical' geometric element is an idealised concept, conveying the idea of an element representative of its class. The typical element cannot be realised exactly, but the sampling of one element (in an equally likely way), from those of that class wholly contained in a large ball $B_{r}$ of radius $r$, approximates the concept and approaches the ideal as $r \rightarrow \infty$. The distribution of the typical element - for example, the length distribution of a typical edge — can be defined by either ergodic theory (see [2] and [3]) or by a formula from the theory of Palm measure (see [11, p. 119]).)

Our results are presented in the isotropic context, but much of the material remains valid for the nonisotropic STIT model. To keep the presentation simple, we do not give the details here of this extended context.

\section{Known results}

An explicit construction of the isotropic STIT tessellation $Y(t)$ on the whole plane has been presented by Mecke et al. [6], [7]. In this construction, the authors go further and construct a stochastic process $(Y(t))_{t>0}$, with $t$ interpreted in two ways: as 'time' and as 'frame intensity'. New $I$-segments are born as time advances, through some divisions of the cells of the tessellation by chords. The particular mechanism for these new chords (described later in this section and in Section 8, in rather different ways, based on the cited papers of Mecke, Nagel and Weiss) ensures that the stochastic structure of $Y(t)$ remains essentially unchanged as time advances, except that the frame intensity increases.

Via the introduction of a planar dilation, the STIT invariance results can be formally written:

$$
\frac{t+\Delta}{t} Y(t+\Delta) \sim Y(t) .
$$

Here $a Y(t)$ is defined as the dilation of $Y(t)$ by a factor $a$ (a point $y \in Y(t)$ is mapped to $a y$ ) and ' $\sim$ ' denotes 'distributed as'. We also note the obvious property that $Y(t) \sim$ STIT implies that $a Y(t) \sim$ STIT with frame intensity $t / a$ for all $t$.

A geometric element appearing in the tessellation has an intensity defined as the mean number of centroids for this class of element per unit area. It is denoted as $\lambda_{X}$, where $X$ is a 
letter indicating the element type. We initially confine our attention to edges $(E)$, sides $(S)$, vertices $(V)$, cells $(Z)$, and $I$-segments $(I)$. We note (see [5]) that a tessellation with frame intensity $t$ has the following intensities:

$$
\lambda_{E}=\frac{3 t^{2}}{\pi}, \quad \lambda_{S}=\frac{4 t^{2}}{\pi}, \quad \lambda_{V}=\frac{2 t^{2}}{\pi}, \quad \lambda_{Z}=\frac{t^{2}}{\pi}, \quad \lambda_{I}=\frac{t^{2}}{\pi} .
$$

\subsection{More recent results from Mecke, Nagel and Weiss}

Mecke, Nagel and Weiss also found (in [7]) a number of distributional results concerning a typical $I$-segment of $Y(t)$. Firstly, they noted that every $I$-segment in $Y(t)$ has a birth time $s \in(0, t)$ and a length $x>0$.

The $I$-segment is born as a new chord dividing a cell $C$ (that existed just prior to time $s$ ) into two daughter cells $C^{+}$and $C^{-}$. The subsequent partitioning of (say) $C^{+}$into smaller cells in the time interval $(s, t]$ involves generating an independent STIT tessellation $Y^{+}(t-s)$ of intensity $t-s$ and filling $\operatorname{Int}\left(C^{+}\right)$with $\operatorname{Int}\left(C^{+}\right) \cap Y^{+}(t-s)$.

Note that the line $\mathcal{L}$ which covers the $I$-segment of $Y(t)$, whilst dependent on $Y(t)$, is not dependent on $Y^{+}(t-s)$. So the transect property, proved in [8], can be employed.

Transect Property. The intersection of the STIT frame $Y(\tau)$ with a reference line $\mathcal{L}$ which is not dependent on $Y(\tau)$ is a stationary Poisson point process $(P P P)$ on $\mathcal{L}$ having intensity $2 \tau / \pi$.

This property with $\tau=t-s$ and $Y$ replaced by $Y^{+}$shows that $\mathcal{L} \cap Y^{+}(t-s)$ is a PPP of intensity $2(t-s) / \pi$. The new vertices on the $I$-segment with edges pointing into $C^{+}$are part of this PPP on $\mathcal{L} \cap Y^{+}(t-s)$. An identical and independent result can be found for those vertices with edges pointing into the other daughter cell, $C^{-}$.

By this method, Mecke et al. showed that the numbers $\left(\xi^{+}\right.$and $\xi^{-}$in their notation) pointing toward $C^{+}$and $C^{-}$, respectively, are conditionally independent given $\{s, x\}$, each with a Poisson $(2 x(t-s) / \pi)$ law. That is, the probability that a typical $I$-segment of $Y(t)$ has $k$ upward-pointing and $j$ downward-pointing internal vertices, given $\{s, x\}$, is

$$
\frac{(2 x(t-s) / \pi)^{k+j}}{k ! j !} \exp \left(-\frac{4}{\pi} x(t-s)\right), \quad k, j=0,1,2, \ldots
$$

Their sum $\xi$ also has the Poisson law, conditionally.

$$
\mathbb{P}\{\xi=m \mid x, s\}=\frac{(4 x(t-s) / \pi)^{m}}{m !} \exp \left(-\frac{4}{\pi} x(t-s)\right), \quad m=0,1,2, \ldots
$$

The joint probability density function of birth time $s$ and length $x$ for the typical $I$-segment was also given in [7]:

$$
\frac{4 s^{2}}{\pi t^{2}} \exp \left(-\frac{2}{\pi} s x\right), \quad 0<s<t, x>0 .
$$

Unconditioning, by using (3), Mecke et al. showed in [7] that, for $k, j, m=0,1,2, \ldots$,

$$
\begin{gathered}
\mathbb{P}\left\{\xi^{+}=k, \xi^{-}=j\right\}=2\left(\begin{array}{c}
k+j \\
j
\end{array}\right) \int_{0}^{1} \frac{a^{k+j}(1-a)^{2}}{(1+a)^{k+j+1}} \mathrm{~d} a, \\
\mathbb{P}\left\{\xi^{+}=k\right\}=\frac{4}{(k+1)(k+2)(k+3)}, \\
p_{m}:=\mathbb{P}\{\xi=m\}=2^{m+1} \int_{0}^{1} \frac{a^{m}(1-a)^{2}}{(1+a)^{m+1}} \mathrm{~d} a .
\end{gathered}
$$


So $p_{0}=8 \log 2-5, p_{1}=2(16 \log 2-11), p_{2}=8(13 \log 2-9), p_{3}=\frac{8}{3}(114 \log 2-79)$, and so on. Also, $\mathbb{E}(\xi)=2$.

Observe that (4) and (5) imply that

$$
\mathbb{P}\left\{\xi^{+}=k \mid \xi=m\right\}=\left(\begin{array}{c}
m \\
k
\end{array}\right)\left(\frac{1}{2}\right)^{m}, \quad k=0,1, \ldots, m,
$$

a fact pointed out in [7].

We note that the numbers of edges and cell sides internal to a typical $I$-segment are $\xi+1$ and $\xi+2$, respectively. So the complete distribution of these entities also follows from (5).

\section{Further structure for the internal vertices}

In 2010, I noticed that the internal vertices of the typical $I$-segment have a more elaborate characterisation, presenting this idea and some of its consequences in a seminar and short note. A proof based on direct integral-geometric calculations with the STIT's cell-division mechanisms (see Section 8) was used.

In this more formal paper, however, I shall derive the characterisation from the results presented in Section 2, specifically from the argument involving two independent PPPs on the line $\mathcal{L}$ which covers the typical $I$-segment of $Y(t)$.

Consider two independent Poisson point processes I and II on $\mathbb{R}$, each with intensity $\theta$. Now superimpose the two processes, creating a marked Poisson process of intensity $2 \theta$ (the marks being I or II). To any reader of this paper, the following two facts are obvious and well known.

- The marks are I or II with probability $\frac{1}{2}$ each, independently for each mark and independent of the process of points.

- Given that there are $m$ points of the superposition process in some interval $[a, b]$, these $m$ points when considered in random order are independently uniformly distributed on $[a, b]$.

So, these facts apply to the internal vertices of the typical $I$-segment, conditional upon $\{s, x\}$ and, for the second fact, with the extra condition $\xi=m$.

Importantly, note that the two facts hold for all values of the intensity $\theta$ and all intervals $[a, b]$. They are independent of $\theta$ and the interval length $b-a$. In our context, this implies that the facts are independent of $s$ and $x$. Therefore, they remain valid after integration over $s$ and $x$, using now the conditional joint probability density function of $x$ and $s$, given $\xi=m$ :

$$
h(x, s \mid m)=\frac{(4 x(t-s) / \pi)^{m}}{p_{m} m !} \frac{4 s^{2}}{\pi t^{2}} \exp \left(-\frac{2}{\pi} x(2 t-s)\right), \quad m=0,1,2, \ldots
$$

These simple observations-which are quite hard to see when the integration is done using (3), yielding a result like (4) —lead to our basic theorem.

Theorem 1. Let $\ell(t)$ be the collection of I-segments in an isotropic, stationary STIT tessellation with frame $Y(t)$, and let $\ell_{m}(t) \subset \ell(t)$ be those that contain $m$ interior vertices. Consider a typical member of $\ell_{m}(t)$, where $m \neq 0$.

(a) The $m$ vertices considered in random order are positioned uniformly and independently along the length of the I-segment.

Each internal vertex is of order three, of course, with one of its three emanating edges not lying in the I-segment. This 'outgoing' edge can be classified as pointing upward (+) from the 
I-segment or downward (-) if one views the segment horizontally.

(b) The choice of ' + ' or '-' is independent for each internal vertex of the I-segment, with probability $\frac{1}{2}$ of being ' + '. In other words, the pointings are determined by independent tosses of a fair coin.

Remark. Theorem 1 is a valuable complement to results (3)-(6) from Mecke et al. [7]. So, when studying a typical $I$-segment, (5) gives us the distribution of $\xi$. The theorem (augmented by the obvious Corollary 1 given below) takes over from there, giving precise positioning and pointing information.

Corollary 1. Consider an experiment where the integer $m$ in Theorem 1 is chosen randomly from a distribution with probabilities $\alpha_{1}, \alpha_{2}, \alpha_{3}, \ldots$ on the support $\{1,2,3, \ldots\}$-and a typical segment of $\ell_{m}(t)$ then chosen. This segment, given $m$, conforms to the theorem. In particular, the typical I-segment, given that it has at least one internal vertex, conforms to the theorem. Furthermore, the I-segment covering a typical edge and the I-segment covering a typical side conform to the theorem (given they have at least one internal vertex).

Proof. The first part of the corollary is obvious and the second part concerning the typical $I$-segment follows with $\alpha_{m}=p_{m} /\left(1-p_{0}\right)$, where $p_{m}$ is given in (5). If $\alpha_{m}=(m+1) p_{m} / \mathbb{E}(\xi+1)=(m+1) p_{m} / 3$ then the sampling scheme gives the properties of the $I$-segment covering the typical edge (since such an $I$-segment has a chance of selection proportional to its number of edges, $m+1$. The one covering the typical side is obtained with $\alpha_{m}=(m+2) p_{m} / \mathbb{E}(\xi+2)=(m+2) p_{m} / 4$.

Corollary 1 is important as it extends Theorem 1, which deals with sampling a typical member of $\ell_{m}(t)$ to a vast number of other sampling schemes for $I$-segments, including all those used later in the paper.

Caveats. Note that (6) does not imply Theorem 1(b), which provides a much more complete understanding of upward or downward pointing of the internal vertices. Nor is the positioning result of Theorem 1(a) a trivial consequence of the transect property of STIT tessellations applied directly to $Y(t)$. The line $\mathcal{L}$ which covers an $I$-segment of $Y(t)$ is dependent on $Y(t)$, so the transect property does not apply in that way. The point process on $\mathcal{L} \cap Y(t)$ is not a Poisson process for such a specially constructed $\mathcal{L}$.

\section{Framework for the calculation}

In the remainder of the paper, we apply Theorem 1 to a detailed study of $I$-segments, sides, and edges. (Terminology: In their work, Mecke et al. referred to 'sides' as $J$-segments and 'edges' as $K$-segments. We consider these labels unnecessary and not as descriptive as the direct (and highly intuitive) geometric labels.)

In the calculations involved, we deal mainly with edges and sides and subtypes of these classes. Typically, we introduce a parameter $\beta$ as the probability that a typical edge (or side) has a certain property $\mathcal{P}$. Defining $\mathcal{P}$ in a given calculation defines a subtype of edge (or side). For example, we might define $\mathcal{P}$ as the edge property 'is an $I$-segment'. Some edges are $I$-segments (and $I$-segments having $\xi=0$ are edges).

Note that $\beta \lambda_{E}$ is the intensity of those edges which have property $\mathcal{P}$. But, this intensity is also given by the expression $\lambda_{I} \mathbb{E} n(\mathcal{P})$, where $n(\mathcal{P})$ is the number of edges lying in the typical 
$I$-segment having the property $\mathcal{P}$. Thus,

$$
\beta=\frac{\lambda_{I}}{\lambda_{E}} \mathbb{E} n(\mathcal{P})=\frac{1}{3} \sum_{m=0}^{\infty} p_{m} \mathbb{E} n(\mathcal{P} \mid m),
$$

where $\mathbb{E} n(\mathcal{P} \mid m)$ is the expectation given that the $I$-segment has $m$ vertices in its relative interior. The intensities $\lambda_{I}$ and $\lambda_{E}$ are given in (1).

Other calculations might involve sides, not edges: for example, define $\mathcal{P}$ as the side property 'contains no internal vertex'. Then we use an identity equivalent to (8) with the notation referring to sides:

$$
\beta=\frac{\lambda_{I}}{\lambda_{S}} \mathbb{E} n(\mathcal{P})=\frac{1}{4} \sum_{m=0}^{\infty} p_{m} \mathbb{E} n(\mathcal{P} \mid m) .
$$

An entity $\beta$ may be difficult to find without Theorem 1 . The essence of our method is to use identities like (8) and (9) and to evaluate the term $\mathbb{E} n(\mathcal{P} \mid m)$ using the power of Theorem 1 and the known probability $p_{m}$ from (5).

\section{Results concerning the typical side}

In some of the evaluations of $\mathbb{E} n(\mathcal{P} \mid m)$ given below, in the context of a 'side property', we use a label attached to each side. Firstly, the $m$ vertices which lie in the relative interior of the $I$-segment (a horizontal one, say) are labelled $1,2, \ldots, m$ from the left to right. In addition, the vertex on the left terminus of the $I$-segment is labelled 0 . A side in the $I$-segment is now labelled according to the label of the vertex on the side's left terminus. Note that two sides are given the label 0 .

Firstly, let us consider some very simple problems.

Problem 1. (Position of the typical side in the covering I-segment.) Let $\mathcal{P}$ be the side property 'is an $I$-segment'. Clearly, using Theorem $1, \mathbb{E} n(\mathcal{P} \mid m)=\left(\frac{1}{2}\right)^{m-1}$. So, the probability that a typical side is an $I$-segment is

$$
\frac{1}{4} \sum_{m=0}^{\infty} p_{m}\left(\frac{1}{2}\right)^{m-1}=\int_{0}^{1}(1-a)^{2} \sum_{m=0}^{\infty} \frac{a^{m}}{(1+a)^{m+1}} \mathrm{~d} a=\int_{0}^{1}(1-a)^{2} \mathrm{~d} a=\frac{1}{3} .
$$

Similar methods complete the following list of results.

- $\mathbb{P}\{$ a typical side is an $I$-segment $\} \equiv \mathbb{P}\{$ a typical side is adjacent to both termini of its covering $I$-segment $\}=\sigma_{2}$ (say) $=\frac{1}{3}$.

- $\sigma_{1}:=\mathbb{P}\{$ a typical side is adjacent to exactly one terminus of its covering $I$-segment $\}=\frac{1}{3}$. Here $\mathbb{E} n(\mathscr{P} \mid m)=2\left(\frac{1}{2}\right)^{m-1}+4\left(1-\left(\frac{1}{2}\right)^{m-1}\right), m \geq 1$, and $\mathbb{E} n(\mathcal{P} \mid 0)=0$.

- $\sigma_{0}:=\mathbb{P}\{$ a typical side is adjacent to neither termini of its covering $I$-segment, that is, it is 'internal' $\}=1-\sigma_{1}-\sigma_{2}=\frac{1}{3}$.

In summary, sides can be classified into three classes according to the number of adjacent termini of its covering $I$-segment. For STIT, these classes are equally likely.

Problem 2. (Internal vertices of a side.) Let $f_{x}$ be defined as the probability that $x$ vertices of the tessellation lie in the relative interior of the typical side. We show that

$$
f_{x}=\int_{0}^{1} \frac{a^{x}}{(1+a)^{x+1}} \mathrm{~d} a, \quad x \geq 0 .
$$


Let $\mathcal{P}_{x}$ be the property 'has $x$ internal vertices'. The calculation of $\mathbb{E} n\left(\mathcal{P}_{x} \mid m\right)$ runs as follows. For $0 \leq m<x, \mathbb{E} n\left(\mathcal{P}_{x} \mid m\right)=0$ whilst, if $m \geq x \geq 0$,

$\mathbb{E} n\left(\mathcal{P}_{x} \mid m\right)=2 \mathbb{P}\{$ a typical side labelled 0 has $x$ internal vertices $\mid m\}$

$$
+\sum_{i=1}^{m-x} \mathbb{P}\{\text { a typical side labelled } i \text { has } x \text { internal vertices } \mid m\},
$$

where the summation term is 0 if $m=x$. The upper bound in the summation is $m-x$ because $\mathbb{P}\{$ side $i$ has $x$ internal vertices $\mid m\}=0$ when $i>m-x$. Furthermore, for $m \geq x \geq 0$,

$\mathbb{P}\{$ a typical side labelled 0 has $x$ internal vertices $\mid m\}= \begin{cases}\left(\frac{1}{2}\right)^{x+1}, & m>x, \\ \left(\frac{1}{2}\right)^{x}, & m=x .\end{cases}$

Also, for $1 \leq i \leq m-x$,

$\mathbb{P}\{$ a typical side labelled $i$ has $x$ internal vertices $\mid m\}= \begin{cases}\left(\frac{1}{2}\right)^{x+1}, & i<m-x, \\ \left(\frac{1}{2}\right)^{x}, & i=m-x .\end{cases}$

Therefore, if $m \geq x \geq 0$,

$$
\mathbb{E} n\left(\mathcal{P}_{x} \mid m\right)= \begin{cases}2\left(\frac{1}{2}\right)^{x}=\left(\frac{1}{2}\right)^{x-1}, & m=x, \\ 2\left(\frac{1}{2}\right)^{x+1}+\left(\frac{1}{2}\right)^{x}+\sum_{i=1}^{m-x-1}\left(\frac{1}{2}\right)^{x+1}=(m-x+3)\left(\frac{1}{2}\right)^{x+1}, & m>x .\end{cases}
$$

So,

$$
\begin{aligned}
f_{x} & =\frac{1}{4} \sum_{m=x}^{\infty} p_{m} \mathbb{E} n\left(\mathcal{P}_{x} \mid m\right) \\
& =\frac{1}{4}\left(\frac{p(x)}{2^{x-1}}+\sum_{m=x+1}^{\infty} p_{m} \frac{m+3-x}{2^{x+1}}\right) \\
& =\frac{1}{4}\left(4 \int_{0}^{1}(1-a)^{2} \frac{a^{x}}{(1+a)^{x+1}} \mathrm{~d} a+\int_{0}^{1}(1-a)^{2} \sum_{m=x+1}^{\infty} 2^{m-x} \frac{(m+3-x) a^{m}}{(1+a)^{m+1}} \mathrm{~d} a\right) \\
& =\frac{1}{4}\left(4 \int_{0}^{1}(1-a)^{2} \frac{a^{x}}{(1+a)^{x+1}} \mathrm{~d} a+4 \int_{0}^{1} \frac{(2-a) a^{x+1}}{(1+a)^{x+1}} \mathrm{~d} a\right) \\
& =\int_{0}^{1} \frac{a^{x}}{(1+a)^{x+1}} \mathrm{~d} a .
\end{aligned}
$$

Note that our expression for $f_{x}$ implies that the mean number of internal vertices in the typical side is $\frac{1}{2}$, a result already known from [6] and available from a general formula for planar tessellations given in [13] (in their notation, $\mu_{Z_{1} V}-2=\phi /\left(\mu_{V E}-\phi\right)=1 /(3-1)=\frac{1}{2}$, evaluated from their Table 4).

Problem 3. (Sides adjacent to a typical side.) In the recent paper of Weiss and Cowan [13], which deals with general planar (and spatial) tessellations, potentially much more complicated than the STIT tessellation, the mean number of sides adjacent to a typical side (denoted by $\mu_{S S}$ ) was not found, though nearly all other adjacencies were expressible in terms of the fundamental parameters of such tessellations. In the STIT model, however, we can now find this mean number $\mu_{S S}$ and, furthermore, we can derive the full distribution of this adjacency variate. 
(The notation of [13] used $\mu_{X Y}$ as the mean number of objects of type $Y$ adjacent to a typical object of type $X$-we conform to this here.)

Let $\mathcal{P}_{x}$ be the property 'is adjacent to $x$ sides'. A side is always adjacent to itself, so $x \geq 1$. The calculation of $\mathbb{E} n\left(\mathcal{P}_{x} \mid m\right)$ follows the method used in the earlier example, using

$\mathbb{E} n\left(\mathcal{P}_{x} \mid m\right)=2 \mathbb{P}\{$ a typical side labelled 0 is adjacent to $x$ sides $\mid m\}$ $+\sum_{i=1}^{m} \mathbb{P}\{$ a typical side labelled $i$ is adjacent to $x$ sides $\mid m\}$.

The situation is quite complicated, but the results are as follows:

$$
\begin{aligned}
& \mathbb{E} n\left(\mathcal{P}_{1} \mid m\right)= \begin{cases}0, & m \leq 2, \\
\frac{1}{4}(m-2), & m \geq 3,\end{cases} \\
& \mathbb{E} n\left(\mathcal{P}_{2} \mid m\right)= \begin{cases}2, & m \leq 1, \\
\frac{7}{2}, & m=2, \\
4+\frac{5}{8}(m-3), & m \geq 3,\end{cases} \\
& \mathbb{E} n\left(\mathcal{P}_{j} \mid m\right)= \begin{cases}\left(\frac{1}{2}\right)^{j-3}, & m=j-2, \\
0, & m=j-1, \quad \text { for } j \geq 3 . \\
\left(\frac{1}{2}\right)^{j-2}, & m=j, \\
\left(\frac{1}{2}\right)^{j-2}+(m-j-1)\left(\frac{1}{2}\right)^{j+1}, & m \geq j+1,\end{cases}
\end{aligned}
$$

Now let $a_{j}$ denote the probability that a typical side is adjacent to $j$ sides. Then

$$
\begin{aligned}
a_{1} & =\frac{1}{4} \sum_{m=3}^{\infty} \frac{m-2}{4} p_{m} \\
& =\frac{1}{8} \int_{0}^{1} \frac{(1-a)^{2}}{1+a} \sum_{m=3}^{\infty}(m-2) \frac{(2 a)^{m}}{(1+a)^{m}} \mathrm{~d} a \\
& =\frac{1}{8} \int_{0}^{1} \frac{a^{3}(1-a)^{2}}{(1+a)^{3}} \mathrm{~d} a \\
& =3 \log 2-2 \\
& \approx 0.07944, \\
a_{2} & =\frac{1}{2} p_{0}+\frac{1}{2} p_{1}+\frac{7}{8} p_{2}+\frac{1}{4} \sum_{m=3}^{\infty}\left[4+\frac{5}{8}(m-3)\right] p_{m} \\
& =1-\frac{1}{2} p_{0}-\frac{1}{2} p_{1}-\frac{1}{8} p_{2}+\frac{5}{32} \sum_{m=3}^{\infty}(m-3) p_{m} \\
& =\frac{47}{2}-33 \log 2+\frac{5}{16} \int_{0}^{1} \frac{(1-a)^{2}}{1+a} \sum_{m=3}^{\infty}(m-3) \frac{(2 a)^{m}}{(1+a)^{m}} \mathrm{~d} a \\
& =\frac{47}{2}-33 \log 2+5 \int_{0}^{1} \frac{a^{4}}{(1+a)^{4}} \mathrm{~d} a \\
& =\frac{23}{8}-3 \log 2 \\
& \approx 0.79556 .
\end{aligned}
$$


For $j \geq 3$,

$$
\begin{aligned}
a_{j} & =\left(\frac{1}{2}\right)^{j}\left[2 p_{j-2}+\sum_{m=j}^{\infty} p_{m}+\frac{1}{8} \sum_{m=j+2}^{\infty}(m-j-1) p_{m}\right] \\
& =\int_{0}^{1} \frac{(1-a)^{2} a^{j-2}}{(1+a)^{j-1}} \mathrm{~d} a+\int_{0}^{1} \frac{2(1-a) a^{j}}{(1+a)^{j}} \mathrm{~d} a+\int_{0}^{1} \frac{a^{j+2}}{(1+a)^{j+1}} \mathrm{~d} a \\
& =\int_{0}^{1} \frac{a^{j-2}}{(1+a)^{j+1}} \mathrm{~d} a .
\end{aligned}
$$

Low moments of a variate $X$ having this probability mass function are as follows:

$$
\begin{aligned}
\mu_{S S} \equiv & \mathbb{E}(X)=\frac{7}{2}-2 \log 2 \approx 2.1137, \\
& \mathbb{E}\left(X^{2}\right)=\frac{21}{2}-8 \log 2 \approx 4.9548, \\
& \mathbb{E}\left(X^{3}\right)=\frac{55}{2}-20 \log 2 \approx 13.6371, \\
& \mathbb{E}\left(X^{4}\right)=\frac{157}{2}-44 \log 2 \approx 48.0015 .
\end{aligned}
$$

\section{A classification of edges}

A companion article to this paper (see [4]) studies general planar tessellations which are not side-to-side. As part of the general theory developed in that article, three parameters, $\epsilon_{0}$, $\epsilon_{1}$, and $\epsilon_{2}$, were introduced. These parameters play a fundamental role in the general theory. They describe the proportions of edges in the tessellation which are equal to 0 , 1, or 2 cell sides. Every edge $e$ is adjacent to two sides — call them $s_{1}$ and $s_{2}$, the sides for which $e \subseteq s_{1}$ and $e \subseteq s_{2}$. Sometimes $e=s_{1}$ or $e=s_{2}$, perhaps both. So, in tessellations which are not side-to-side, an edge $e$ can equal either 0,1 , or 2 sides.

The probability that a typical edge equals $j$ sides (call this a type- $j$ edge) is denoted by $\epsilon_{j}$, $j=0,1,2$, where $\epsilon_{0}+\epsilon_{1}+\epsilon_{2}=1$. In this section we evaluate these three parameters for the STIT tessellation.

An edge in the STIT tessellation equals two sides, if and only if it is an $I$-segment. So, given that an $I$-segment comprises only one edge, an event with probability $p_{0}=8 \log 2-5$ as given in (5), this edge is of type 2. Also, given that an $I$-segment comprises two edges, both are of type 1.

For the typical $I$-segment, let $\zeta_{i}$ be the number of edges of type $i$.

Corollary 2. Given that a typical I-segment has $j \geq 3$ edges, then $\zeta_{0}+\zeta_{1}=j$ and $\zeta_{0}$ is distributed as Binomial $\left[j-2, \frac{1}{2}\right]$, as is $\zeta_{1}-2$. The statement holds for $j=2$ also, in a degenerate sense.

Proof. Consider the $I$-segment in a horizontal position. With $j \geq 3$ edges (and hence $j-1$ internal vertices and $j-2$ internal edges), the edge adjacent to the left terminus must be of type 1 . Theorem 1 states that the pointings of the remaining $j-2$ internal vertices are determined as ' + ' or ' - ' by independent coin tosses. Another description instead of ' + ' or ' - ' is 'same' or 'different' to the pointing immediately to the left. These descriptors too are independent Bernoulli variates, parameter $\frac{1}{2}$. The number of 'differents' is therefore $\operatorname{Binomial}\left[j-2, \frac{1}{2}\right]$. But an internal edge whose right terminus hosts a 'different' pointing is of type 0 . Therefore, $\zeta_{0} \sim \operatorname{Binomial}\left[j-2, \frac{1}{2}\right]$. An internal edge whose right terminus hosts a 'same' pointing is of type 1 . So, $\zeta_{1}-2 \sim \operatorname{Binomial}\left[j-2, \frac{1}{2}\right]$, the subtraction of 2 being because both noninternal edges must be of type 1 . 
Let $q\left(z_{0}, z_{1}\right)$ be the probability that, for a typical $I$-segment, $\zeta_{0}=z_{0}$ and $\zeta_{1}=z_{1}$. Then

$$
q\left(z_{0}, z_{1}\right)= \begin{cases}p_{0}, & z_{0}=z_{1}=0 \\
p_{z_{0}+z_{1}-1}\left(\begin{array}{c}
z_{0}+z_{1}-2 \\
z_{0}
\end{array}\right)\left(\frac{1}{2}\right)^{z_{0}+z_{1}-2}, & z_{1} \geq 2 \\
0, & \text { otherwise }\end{cases}
$$

\subsection{The distribution of $\zeta_{0}$}

The marginal probability mass function, $q_{0}$ say, of $\zeta_{0}$ in a typical $I$-segment is given by the following formulae:

$$
\begin{gathered}
q_{0}(0)=p_{0}+\sum_{z_{1}=2}^{\infty} p_{z_{1}-1}\left(\begin{array}{c}
z_{1}-2 \\
0
\end{array}\right)\left(\frac{1}{2}\right)^{z_{1}-2}, \\
q_{0}\left(z_{0}\right)=\sum_{z_{1}=2}^{\infty} p_{z_{0}+z_{1}-1}\left(\begin{array}{c}
z_{0}+z_{1}-2 \\
z_{0}
\end{array}\right)\left(\frac{1}{2}\right)^{z_{0}+z_{1}-2}, \quad z_{0} \geq 1 .
\end{gathered}
$$

For $z_{0} \geq 1$,

$$
\begin{aligned}
q_{0}\left(z_{0}\right) & =\sum_{z_{1}=2}^{\infty}\left(\begin{array}{c}
z_{0}+z_{1}-2 \\
z_{0}
\end{array}\right)\left(\frac{1}{2}\right)^{z_{0}+z_{1}-2} \times 2^{z_{0}+z_{1}} \int_{0}^{1}(1-a)^{2} \frac{a^{z_{0}+z_{1}-1}}{(1+a)^{z_{0}+z_{1}}} \mathrm{~d} a \\
& =4 \int_{0}^{1}(1-a)^{2}\left[\sum_{z_{1}=2}^{\infty}\left(\begin{array}{c}
z_{0}+z_{1}-2 \\
z_{0}
\end{array}\right) \frac{a^{z_{0}+z_{1}-1}}{(1+a)^{z_{0}+z_{1}}}\right] \mathrm{d} a \\
& =4 \int_{0}^{1}(1-a)^{2} \frac{a^{z_{0}-1}}{(1+a)^{z_{0}}}\left(\frac{a}{1+a}\right)^{2} \frac{1}{(1-a /(1+a))^{z_{0}+1}} \mathrm{~d} a \\
& =4 \int_{0}^{1} \frac{(1-a)^{2} a^{z_{0}+1}}{1+a} \mathrm{~d} a \\
& =\frac{8}{\left(z_{0}+2\right)\left(z_{0}+3\right)\left(z_{0}+4\right)}{ }_{2}^{2} F_{1}\left(1, z_{0}+2 ; z_{0}+5 ;-1\right) .
\end{aligned}
$$

Here, ${ }_{2} F_{1}$ is the generalised hypergeometric function (see [1, p. 556]). It is finite in the current context because ${ }_{2} F_{1}(a, b ; c ;-1)$ is an absolutely convergent series when $c-a-b>0$, as is the case here. This calculation also gives us

$$
\begin{aligned}
q_{0}(0) & =p_{0}+4 \int_{0}^{1} \frac{(1-a)^{2} a}{1+a} \mathrm{~d} a \\
& =(8 \log 2-5)+\frac{2}{3}(17-24 \log 2) \\
& =\frac{19}{3}-8 \log 2 \\
& \approx 0.7882 .
\end{aligned}
$$

The expectation of $\zeta_{0}$ is

$$
\mathbb{E}\left(\zeta_{0}\right)=4 \int_{0}^{1} \frac{(1-a)^{2}}{1+a} \sum_{z_{0}=1}^{\infty} z_{0} a^{z_{0}+1} \mathrm{~d} a=4 \int_{0}^{1} \frac{a^{2}}{1+a} \mathrm{~d} a=2(2 \log 2-1) .
$$




\subsection{The distribution of $\zeta_{1}$}

The marginal probability mass function of $\zeta_{1}$ is, for $z_{1} \geq 2$,

$$
q_{1}\left(z_{1}\right)=4 \int_{0}^{1} \frac{(1-a)^{2} a^{z_{1}-1}}{1+a} \mathrm{~d} a=\frac{8}{z_{1}\left(z_{1}+1\right)\left(z_{1}+2\right)} 2 F_{1}\left(1, z_{1} ; z_{1}+3 ;-1\right)
$$

and

$$
\mathbb{E}\left(\zeta_{1}\right)=4 \int_{0}^{1} \frac{(1-a)^{2}}{1+a} \sum_{z_{1}=2}^{\infty} z_{1} a^{z_{1}-1} \mathrm{~d} a=4 \int_{0}^{1} \frac{a(2-a)}{1+a} \mathrm{~d} a=2(5-6 \log 2) .
$$

Since $\mathbb{E}\left(\zeta_{2}\right)=8 \log 2-5$, we have the expected total count of all three types as

$$
2(2 \log 2-1)+2(5-6 \log 2)+(8 \log 2-5)=3,
$$

as required. (Since $\mathbb{E} \xi=2$, the expected number of edges in a typical $I$-segment is 3 .)

\subsection{The epsilons}

We now evaluate the probabilities $\epsilon_{0}, \epsilon_{1}$, and $\epsilon_{2}$ for the STIT model. Let us denote the class of $j$-type edges by $E[j]$ and the intensity of this type by $\lambda_{E[j]}$. Clearly, $\lambda_{E[j]}=\lambda_{I} \mathbb{E}\left(\zeta_{j}\right)=$ $\frac{1}{3} \lambda_{E} \mathbb{E}\left(\zeta_{j}\right)$. Therefore,

$$
\begin{aligned}
& \epsilon_{2}=\frac{\lambda_{E[2]}}{\lambda_{E}}=\frac{1}{3}(8 \log 2-5) \approx 0.18173, \\
& \epsilon_{1}=\frac{\lambda_{E[1]}}{\lambda_{E}}=\frac{2}{3}(5-6 \log 2) \approx 0.56075, \\
& \epsilon_{0}=\frac{\lambda_{E[0]}}{\lambda_{E}}=\frac{2}{3}(2 \log 2-1) \approx 0.25753 .
\end{aligned}
$$

\section{Metric properties}

In Sections 5 and 6 we focused on topological issues. In this section, our concerns are metric in character, focusing on lengths of various line segments and on frame coverage. We also give results concerning the birth time of certain segments, within the temporal framework of [7]. Another corollary to Theorem 1 is used in obtaining some of the metric results.

Corollary 3. Given that a typical segment belonging to $\ell_{m}(t), m \neq 0$, is of length $x$, the length $Y$ of an arbitrarily selected edge within the segment has probability density function $m x^{-1}(1-y / x)^{m-1}, 0<y<x$. This is true regardless of the edge type, 0 or 1 .

Proof. The proof follows from the well-known spacing properties of $m \geq 1$ uniformly distributed and independent variates in the interval $(0, x)$.

We commence the section with a review of known results for the lengths of $I$-segments, edges, and sides. Then we make our first use of Corollary 3.

Known length distributions. The probability density functions of lengths for typical $I$-segments, sides, and edges of $Y(t)$ have been found in [5]; see also [7]. These are

$$
\begin{aligned}
& f_{I}(x)=\frac{1}{t^{2} x^{3}}\left(\pi^{2}-\left(\pi^{2}+2 \pi t x+2 t^{2} x^{2}\right) \mathrm{e}^{-2 t x / \pi}\right), \quad f_{S}(x)=\frac{2 t}{\pi} \mathrm{e}^{-2 t x / \pi}, \\
& f_{E}(x)=\frac{1}{3 t^{2} x^{3}}\left(\left(\pi^{2}+2 \pi t x+2 t^{2} x^{2}\right) \mathrm{e}^{-2 t x / \pi}-\left(\pi^{2}+4 \pi t x+8 t^{2} x^{2}\right) \mathrm{e}^{-4 t x / \pi}\right) .
\end{aligned}
$$

The expected lengths are $\pi / t$ for $I, \pi / 2 t$ for $S$, and $\pi / 3 t$ for $E$. Note that $f_{I}$ can also be found from (3) by integrating over $s$. 
TABLE 1.

\begin{tabular}{rc}
\hline$m$ & $t \mathbb{E}(Y \mid m) / \pi$ \\
\hline 0 & 0.417 \\
1 & 0.379 \\
2 & 0.357 \\
5 & 0.322 \\
10 & 0.297 \\
20 & 0.279 \\
\hline
\end{tabular}

The length of a random edge of a typical I-segment. Sometimes sampling is a twostage process. Here we sample a typical $I$-segment, then randomly choose one of its edges. Corollary 3 is obviously relevant and, from it, one can immediately write down the probability density function of $Y$, the length of the random edge (using entities defined in (5) and (7)):

$$
\begin{gathered}
p_{0} \int_{0}^{t} h(y, s \mid 0) \mathrm{d} s+\sum_{m=1}^{\infty} p_{m} \int_{y}^{\infty}\left(\int_{0}^{t} h(x, s \mid m) \mathrm{d} s\right) \frac{m}{x}\left(1-\frac{y}{x}\right)^{m-1} \mathrm{~d} x \\
=\frac{1}{t^{2} y^{3}}\left(\left(2 t^{2} y^{2}-\pi^{2}\right) \mathrm{e}^{-2 t x / \pi}+\left(\pi^{2}+2 \pi t y\right) \mathrm{e}^{-4 t x / \pi}\right) .
\end{gathered}
$$

The mean length is $(2 \log 2-1) \pi / t(\approx 0.3863 \pi / t)$, which is sensibly greater than the mean length of the typical edge, $\pi / 3 t$. This method of sampling has a bias against edges in $I$-segments with many edges, and $\mathbb{E}(Y \mid m)$ decreases with $m$, as the numerical results in Table 1 show.

Note that analytic expressions are available for every entry in Table 1. For example,

$$
\begin{aligned}
\mathbb{E}(Y \mid m) & =\int_{0}^{\infty} y \int_{0}^{t} \int_{y}^{\infty} h(x, s \mid m) \frac{m}{p_{m} x}\left(1-\frac{y}{x}\right)^{m-1} \mathrm{~d} x \mathrm{~d} s \mathrm{~d} y \\
& =\frac{2^{m} \pi}{p_{m} t^{2}} \int_{0}^{t} \frac{s^{2}(t-s)^{m}}{(2 t-s)^{m+2}} \mathrm{~d} s \\
& =\frac{\pi(5989-8640 \log 2)}{64 t(1020 \log 2-707)} \quad \text { if (say) } m=5 .
\end{aligned}
$$

The I-segment covering a typical edge. An I-segment's chance of being chosen, when one samples first a typical edge and then the $I$-segment which covers that edge, is proportional to the number of edges within the $I$-segment. So it will contain $m$ internal vertices (hence, $m+1$ edges) with probability $q_{m}:=(m+1) p_{m} /(\mathbb{E}(\xi)+1)=\frac{1}{3}(m+1) p_{m}$. Furthermore, the conditional joint probability density function of segment length $x$ and birth time $s$ given $m$ remains as $h(x, s \mid m)$ (see (7)). Thus, the joint probability density function of segment length $x$ and birth time $s$ is (for $x>0$ and $0<s<t$ )

$$
\begin{aligned}
\sum_{m=0}^{\infty} h(x, s \mid m) q_{m} & =\sum_{m=0}^{\infty} \frac{(4 x(t-s) / \pi)^{m}}{p_{m} m !} \frac{4 s^{2}}{\pi t^{2}} \exp \left(-\frac{2}{\pi} x(2 t-s)\right) \frac{(m+1) p_{m}}{3} \\
& =\frac{4 s^{2}}{3 \pi t^{2}} \exp \left(-\frac{2}{\pi} x(2 t-s)\right) \sum_{m=0}^{\infty} \frac{(4 x(t-s) / \pi)^{m}}{m !}(m+1) \\
& =\frac{4 s^{2}}{3 \pi t^{2}}\left(1+\frac{4}{\pi} x(t-s)\right) \mathrm{e}^{-2 s x / \pi} .
\end{aligned}
$$


TABLE 2.

\begin{tabular}{ccccc}
\hline \multirow{2}{*}{ Type, $j$} & \multicolumn{4}{c}{$q_{m}$} \\
\cline { 2 - 5 } & $m \geq 2$ & $m=1$ & $m \geq 1$ & $m=0$ \\
\hline 0 & $\frac{(m-1) p_{m}}{6 \epsilon_{0}}$ & 0 & & 0 \\
1 & & & $\frac{(m+3) p_{m}}{6 \epsilon_{1}}$ & 0 \\
2 & & & 0 & 1 \\
\hline
\end{tabular}

From this, the marginal densities for birth time $s$ and length $x$ are respectively

$$
\frac{2(2 t-s)}{3 t^{2}}, \quad 0<s<t ; \quad \frac{\left(5 \pi^{2}+6 \pi t x+2 t^{2} x^{2}\right) \mathrm{e}^{-2 t x / \pi}+4 \pi t x-5 \pi^{2}}{3 t^{2} x^{3}}, \quad x>0 .
$$

Note that the expected length is infinite.

A random edge of the I-segment covering a typical edge. Once again we use Corollary 3. The length $Y$ of such an edge has probability density function given by

$$
q_{0} \int_{0}^{t} h(y, s \mid 0) \mathrm{d} s+\sum_{m=1}^{\infty} q_{m} \int_{y}^{\infty}\left(\int_{0}^{t} h(x, s \mid m) \mathrm{d} s\right) \frac{m}{x}\left(1-\frac{y}{x}\right)^{m-1} \mathrm{~d} x=f_{E}(y) .
$$

It does not come as a surprise that a random edge of the $I$-segment covering a typical edge is also typical!

The I-segment covering a typical side. The analysis follows the section above, except that now $q_{m}:=(m+2) p_{m} /(\mathbb{E}(\xi)+2)=\frac{1}{4}(m+2) p_{m}$. The joint probability density function of segment length $x$ and birth time $s$ is (for $0<s<t$ and $x>0$ )

$$
\frac{2 s^{2}}{\pi t^{2}}\left(1+\frac{2}{\pi} x(t-s)\right) \mathrm{e}^{-2 s x / \pi}
$$

whilst the marginals are

$$
\frac{1}{t}, \quad 0<s<t ; \quad \pi \frac{(\pi+t x) \mathrm{e}^{-2 t x / \pi}+t x-\pi}{t^{2} x^{3}}, \quad x>0 .
$$

The expected length is again infinite. Note the uniformly distributed birth time!

The I-segment covering a typical type- $j$ edge. Now $q_{m}$ takes the form shown in Table 2.

The joint probability density function of segment length $x$ and birth time $s$ is (for $0<s<t$ and $x>0$ )

$$
\begin{array}{cc}
\frac{s^{2}\left(\pi \mathrm{e}^{-2(2 t-s) x / \pi}-(\pi-4 x(t-s)) \mathrm{e}^{-2 s x / \pi}\right)}{\pi^{2} t^{2}(2 \log 2-1)} & \text { for } j=0, \\
\frac{s^{2}\left((3 \pi+4 x(t-s)) \mathrm{e}^{-2 s x / \pi}-3 \mathrm{e}^{-2(2 t-s) x / \pi}\right)}{\pi^{2} t^{2}(5-6 \log 2)} & \text { for } j=1, \\
\frac{4 s^{2} \mathrm{e}^{-2(2 t-s) x / \pi}}{\pi t^{2}(8 \log 2-5)} \text { for } j=2 . &
\end{array}
$$


TABLE 3.

\begin{tabular}{lcc}
\hline$j$ & \multicolumn{2}{c}{ Probability density function } \\
\cline { 2 - 3 } & Birth time, $s$ & Length, $x$ \\
\hline 0 & $\frac{2(t-s)^{2}}{(2 \log 2-1)(2 t-s) t^{2}}$ & $\pi \frac{4 t x-7 \pi+8\left(\pi+t x+t^{2} x^{2} / \pi\right) \mathrm{e}^{-2 t x / \pi}-\pi \mathrm{e}^{-4 t x / \pi}}{4 t^{2} x^{3}(2 \log 2-1)}$ \\
1 & $\frac{2\left(t^{2}-s^{2}\right)}{(5-6 \log 2)(2 t-s) t^{2}}$ & $\frac{4 \pi t x-3 \pi^{2}+8 t x(\pi-t x) \mathrm{e}^{-2 t x / \pi}+3 \pi^{2} \mathrm{e}^{-4 t x / \pi}}{4 t^{2} x^{3}(5-6 \log 2)}$ \\
2 & $\frac{2 s^{2}}{(8 \log 2-5)(2 t-s) t^{2}}$ & $\frac{\left(\pi^{2}-2 \pi t x+2 t^{2} x^{2}\right) \mathrm{e}^{-2 t x / \pi}-\pi^{2} \mathrm{e}^{-4 t x / \pi}}{t^{2} x^{3}(8 \log 2-5)}$ \\
\hline
\end{tabular}

We note that the $(j=2)$ length distribution agrees with the length distribution of $I$-segments which contain no internal vertices, as it obviously should. Using (2) and (3), this is

$$
\begin{aligned}
f_{I}(x \mid \xi=0) & =\frac{1}{\mathbb{P}\{\xi=0\}} \int_{0}^{t} h(x, s \mid 0) \mathrm{d} s \\
& =\frac{1}{t^{2} x^{3}(8 \log 2-5)}\left(\left(\pi^{2}-2 \pi t x+2 t^{2} x^{2}\right) \mathrm{e}^{-2 t x / \pi}-\pi^{2} \mathrm{e}^{-4 t x / \pi}\right)
\end{aligned}
$$

which has mean $\pi(3-4 \log 2) /[t(8 \log 2-5)] \approx 0.4171 \pi / t$. This is the only finite expected length in Table 3.

Length of type- $j$ edges, $j=0,1,2$. The distribution in (10) also describes the length of edges that are equal to two sides (type- 2 edges, or those whose configuration looks like $\longmapsto$ if viewed horizontally) because the class of edges equal to two sides and the class of $I$-segments with $\xi=0$ are equivalent. So, $f_{E[2]}(y)=f_{I}(y \mid \xi=0)$.

We show, in the now familiar way which depends on Corollary 2 , that the probability density function of length $Y$ for type-0 edges (those configured horizontally like $\perp_{T_{1}}$ or $\frac{\perp}{\Gamma}$ ) is

$$
f_{E[0]}(y)=\frac{1}{t^{2} y^{3}(2 \log 2-1)}\left(\pi^{2} \mathrm{e}^{-2 t y / \pi}-\left(\pi^{2}+2 \pi t y+2 t^{2} y^{2}\right) \mathrm{e}^{-4 t y / \pi}\right) .
$$

Using $q_{m}:=(m-1) p_{m} / 6 \epsilon_{0}$, the probability density function of $Y$ is

$$
q_{0} \int_{0}^{t} h(y, s \mid 0) \mathrm{d} s+\sum_{m=1}^{\infty} q_{m} \int_{y}^{\infty}\left(\int_{0}^{t} h(x, s \mid m) \mathrm{d} s\right) \frac{m}{x}\left(1-\frac{y}{x}\right)^{m-1} \mathrm{~d} x,
$$

which reduces to the form $f_{E[0]}(y)$ in (11). The expected length $\mathbb{E}(Y)$ is given by $\pi(3-$ $4 \log 2) /(2 t(2 \log 2-1))=0.2944 \pi / t$.

By a similar method, or by combining this result with earlier results for $f_{E}(\cdot)$ and $f_{E[2]}(\cdot)$, we can show that, for the type-1 edge ( $\uparrow, \perp, \uparrow, \downarrow, \perp, \perp$, or $T \top)$,

$$
\begin{aligned}
f_{E[1]}(y) & =\frac{1}{\epsilon_{1}}\left(f_{E}(y)-\epsilon_{0} f_{E[0]}(y)-\epsilon_{2} f_{E[2]}(x)\right) \\
& =\frac{1}{t^{2} y^{3}(5-6 \log 2)}\left(\left(2 \pi t y-\pi^{2}\right) \mathrm{e}^{-2 t y / \pi}+\left(\pi^{2}-2 t^{2} y^{2}\right) \mathrm{e}^{-4 t y / \pi}\right) .
\end{aligned}
$$

The expected length is $\pi(8 \log 2-5) /(2 t(5-6 \log 2))$, or $0.3241 \pi / t$. 
Frame coverage. The length intensity of type- 2 edges (defined as the expected length per unit area) is obviously $\lambda_{E} \epsilon_{2}$ times the mean calculated from (10), or

$$
\frac{3 t^{2}}{\pi} \frac{8 \log 2-5}{3} \frac{\pi(3-4 \log 2)}{t(8 \log 2-5)}=t(3-4 \log 2) \approx 0.2274 t .
$$

This means that $22.74 \%$ of the frame is covered by type- 2 edges (or, equivalently, by $I$-segments with $\xi=0)$. Similar calculations show that the same proportion $(22.74 \%)$ of the frame is covered by type- 0 edges, leaving a proportion $8 \log 2-5$ (or $54.52 \%$ ) covered by type- 1 edges.

Furthermore, the type-1 proportion comprises two parts:

- $22.74 \%$ of the frame is covered by type- 1 edges not adjacent to an end of the $I$-segment $(\perp$ or $T \mathrm{~T})$;

- $31.78 \%$ of the frame is covered by type- 1 edges which are adjacent to an end of the $I$-segment $\left(\uparrow, \stackrel{\perp}{\uparrow}, \uparrow_{\uparrow}\right.$, or $\downarrow$ - $)$.

\section{Vertices ordered by the birth time}

In Section 5 we labelled the internal vertices of an $I$-segment by $1,2, \ldots, m$ as we move along the segment. In Theorem 1 we considered these $m$ vertices in random order, specifically an order that is a permutation of $\{1,2, \ldots, m\}$ with every permutation being equally likely. There is another approach, however. It uses the fact that internal vertices of the $I$-segment have birth times.

Theorem 2. The results of Theorem 1 and its corollaries are valid if the order of the $m$ vertices is that of their birth times.

As background for a proof of Theorem 2, we shall describe the STIT cell division process in greater detail, giving the random-geometric evolution of the interior of a convex cell $C$, extant at time $t$. A lifetime $L$ for $C$ is sampled from an exponential distribution with mean inversely proportional to the cell's perimeter, that is, $\mathbb{P}\{L \leq u\}=1-\exp (-\lambda \operatorname{Perim}(C) u)$. At time $t+L$, a uniformly random (UR) chord of $C$ is constructed, dividing $C$ into two daughter cells. This chord is an $I$-segment. (UR means that each chord of $C$ is equally likely to be chosen.)

For each daughter, independent lifetimes are sampled using the same protocol (with the perimeters of the daughters determining the means of the exponential variates). A daughter is divided by a UR chord when its lifetime ends. This construction continues for all cells created within cell $C$.

Proof of Theorem 2. Consider at time $t$ any $I$-segment. Call it $I$. Suppose that $I$ has $k \geq 0$ internal vertices and, therefore, $k+1$ edges. Also, note that there are $k+2$ cells of the tessellation which have a side in $I$. Call these the adjoining cells. If $I$ is viewed vertically, suppose that $n$ of these cells are to the left of $I$ having side lengths $\ell_{1}, \ell_{2}, \ldots, \ell_{n}$ lying along $I$. Thus, $k+2-n$ are to the right; denote their side lengths by $r_{1}, r_{2}, \ldots, r_{k+2-n}$. The 'left' cells have perimeters $L_{1}, L_{2}, \ldots, L_{n}$ and 'right' cells have perimeters $R_{1}, R_{2}, \ldots, R_{k+2-n}$. Obviously, $\sum_{i} \ell_{i}=\sum_{i} r_{i}=|I|$, the length of $I$.

We observe $Y(t+\tau)$, with $\tau$ increasing continuously, until a new chord hits $I$, creating a new internal vertex of the segment. The opportunity to create a new internal vertex occurs whenever an adjoining cell divides; if the cell dividing is (say) the ' $\operatorname{left}(j)$ cell', the one with perimeter $L_{j}$ and side-in- $I \ell_{j}$, there is a chance $2 \ell_{j} / L_{j}$ that its new UR chord will hit $I$. If it 
does so, the hitting point will be uniformly distributed on the left $(j)$-cell's side within $I$. These facts come from well-known properties of UR chords of convex domains (see [10]).

The left $(j)$ cell will, with probability $L_{j} /\left(\sum_{i} L_{i}+\sum_{i} R_{i}\right)$, be the first of the adjoining cells to divide after time $t$ (a property of independent exponential variates). So this cell has a chance

$$
\frac{L_{j}}{\sum_{i} L_{i}+\sum_{i} R_{i}} \frac{2 \ell_{j}}{L_{j}}=\frac{2 \ell_{j}}{\sum_{i} L_{i}+\sum_{i} R_{i}}
$$

to achieve the hit of $I$ at this opportunity. Therefore, the probability that a left cell divides first and its chord creates a hit is $\left(\sum_{j} 2 \ell_{j}\right) /\left(\sum_{i} L_{i}+\sum_{i} R_{i}\right)$, which equals $2|I| /\left(\sum_{i} L_{i}+\sum_{i} R_{i}\right)$.

Likewise, the probability that a right cell is first to divide and it's chord creates a hit is also $2|I| /\left(\sum_{i} L_{i}+\sum_{i} R_{i}\right)$. So, if a hit occurs from the first division of an adjoining cell then, independently of the geometry of adjoining cells,

- the hit is equally likely to be from the left or right;

- the position of the hit is uniformly distributed within the dividing cell's $I$-touching side;

- an adjoining cell's chance of being the cell whose chord hits $I$ is proportional to the length of its $I$-touching side.

The last two bullet points imply that the position of the new internal vertex is uniformly distributed on $I$, independently of the number and positioning of the internal vertices of $I$, extant at time $t$.

If the first cell division amongst the adjoining cells does not create a new internal vertex in $I$, the number of adjoining cells remains at $k+2$, with $n$ on the left and $k+2-n$ on the right, as initially. The only change from the starting situation is that one of the original adjoining cells has been divided (with a chord that did not hit $I$ ). So we just replace that cell with its daughter that has contact with $I$. We merely transfer cell labels and variables, for example, if the dividing cell was 'left $(j)$ ', its $I$-touching daughter takes over the label 'left $(j)$ ' and ownership of the variates $L_{j}$ (whose value has changed) and $\ell_{j}$.

We now start the argument again. Eventually, $I$ will be hit and, when that hit occurs, the bullet-point statements will apply (but now, independently of the most recent geometry of the adjoining cells and their original geometry at time $\tau$ ).

This completes the proof that the first new internal vertex on $I$ has the properties given in the theorem. To deal with the second such vertex, we merely repeat the argument (observing the process as $\tau$ increases). Likewise for the third vertex and later vertices.

So we have established that all new vertices created in the post- $t$ cell division operations are positioned and pointed in the manner described by the theorem. Indeed, by setting $k=0$ in the argument above, we describe the evolution of $I$ from the moment of its birth. All of the internal vertices it acquires are independently uniformly distributed on the segment's length, independently of past geometry, and their pointings are determined by coin tossing.

\section{Conclusion}

Nearly all the results presented in this paper are new, and most of them are derived via an application of Theorem 1. This theorem, although simply proved via a novel interpretation and extension of results in [7], is therefore a very useful characterisation of the internal structure of STIT's $I$-segments. The results might also have been derived in the 'temporal ordering' context of Theorem 2 . 


\section{References}

[1] Abramowitz, M. And Stegun, I. A. (1970). Handbook of Mathematical Functions. Dover Publications, New York.

[2] Cowan, R. (1978). The use of ergodic theorems in random geometry. Suppl. Adv. Appl. Prob. 10, 47-57.

[3] Cowan, R. (1980). Properties of ergodic random mosaic processes. Math. Nachr. 97, 89-102.

[4] Cowan, R. and Thäle, C. (2013). The character of planar tessellations which are not side-to-side. Submitted.

[5] Mecke, J., Nagel, W. and Weiss, V. (2007). Length distributions of edges in planar stationary and isotropic STIT tessellations. Izv. Akad. Nauk Armenii Mat. 42, 39-60. English translation: J. Contemp. Math. Anal. 42, $28-43$.

[6] Mecke, J., Nagel, W. and Weiss, V. (2008). A global construction of homogeneous random planar tessellations that are stable under iteration. Stochastics 80, 51-67.

[7] Mecke, J., NAGEL, W. AND Weiss, V. (2011). Some distributions for I-segments of planar random homogeneous STIT tessellations. Math. Nachr. 284, 1483-1495.

[8] Nagel, W. and Weiss, V. (2003). Limits of sequences of stationary planar tessellations. Adv. Appl. Prob. 35, $123-138$.

[9] NAGEL, W. AND WEISs, V. (2005). Crack STIT tessellations: characterization of stationary random tessellations stable with respect to iteration. Adv. Appl. Prob. 37, 859-883.

[10] Santaló, L. A. (1976). Integral Geometry and Geometric Probability (Encyclopedia Math. Appl. 1). Addison Wesley, Reading, MA.

[11] Stoyan, D., Kendall, W. S. And Mecke, J. (1995). Stochastic Geometry and Its Applications, 2nd edn. John Wiley, Chichester.

[12] THÄLE, C. (2010). The distribution of the number of nodes in the relative interior of the typical I-segment in homogeneous planar anisotropic STIT tessellations. Comment. Math. Univ. Carolin. 51, 503-512.

[13] Weiss, V. And Cowan, R. (2011). Topological relationships in spatial tessellations. Adv. Appl. Prob. 43,963-984.

[14] Weiss, V., Ohser, J. And Nagel, W. (2010). Second moment measure and $K$-function for planar STIT tessellations. Image Anal. Stereol. 29, 121-131. 